\title{
Correspondence
}

\section{Eating disorder in children and adolescents - risky business?}

Although we agree with Dr Wrate that a context to risk is important, ${ }^{1}$ we cannot concur with his view of the context. As healthcare professionals caring for young people with eating disorders, we do not regard seven deaths in children and adolescents as reassuring. When put into the context of more 'traditional' paediatric illnesses, 1.5 deaths per year are not far removed from 12 deaths from diabetic ketoacidosis and 14 deaths from invasive childhood meningococcal disease (sources available from the authors on request). Even within the 18 years since Dr Wrate's search, there has been a continuing reduction of vaccine-preventable diseases, improvement in neonatal outcomes and survival from childhood malignancies, resulting in a shift in childhood illnesses from communicable to non-communicable diseases, including eating disorders.

Death is a preventable outcome of eating disorders and may be secondary to lack of awareness, knowledge or timely treatment; yet it would seem that paediatric medical and mental health services are sometimes ill equipped to meet the challenges of young patients presenting with eating disorders, especially acutely. Studies show that $50 \%$ of children less than 13 years old are hospitalised early in their illness ${ }^{2}$ and numbers of children under 14 hospitalised for eating disorders have risen over the past 10 years. A recent survey of on-call paediatric registrars in hospitals in England and Wales revealed poor knowledge of the acute management of children with eating disorders (C. Cumby, 2012, personal communication). Most were unaware of the frequent cardiovascular complications and unable to outline the complications of refeeding syndrome.

We agree with Dr Wrate that there has been too much emphasis historically on hospitalisation rather than on goodquality out-patient treatments and early intervention; however, we believe that it is important not to negate risk in order to redress this balance. The decision to admit is a complex one; unfortunately, it is our observation that admission to hospital is often more dependent on available services and experience of healthcare professionals than clinical need.

We also wish to comment on Dr Wrate's commentary on morbidity in eating disorders in childhood, which he describes as relating only to bone mineral density. Not wanting to understate the significance of potentially irreversible low bone density as a medical complication, we must highlight the multisystem effects of eating disorders and associated malnutrition in this group. Other unique medical complications found in children with eating disorders include an impact on growth and development and changes in brain structure and function. ${ }^{3,4}$ Such complications are treatable if recognised early and treated aggressively.

To date, there are too few data on the outcomes of children with eating disorders for us to relax about risk. In preparing the Junior MARSIPAN report, ${ }^{5}$ instances of deaths were rare. Nonetheless, the cases described in the report show this is risky business indeed and examples were not hard to come by. The risk assessment framework in the Junior
MARSIPAN report acknowledges the complexity of risk assessment, and is stratified to avoid overstatement. Valuable lessons learnt from improvements in survival from preventable childhood diseases such as meningococcal disease include the importance of early recognition, awareness and training. In the absence of consistency, we argue that erring on the side of caution is prudent and the MARSIPAN guidelines are important safeguards to help clinicians understand, recognise and treat eating disorders.

\section{Declaration of interest}

L.H. and D.N. contributed as authors to Junior MARSIPAN; D.N. is president of the Academy for Eating Disorders.

1 Wrate RM. Death and risk in adolescent anorexia nervosa. Psychiatrist 2012; 36: 316-7.

2 Nicholls DE, Lynn R, Viner RM. Childhood eating disorders: British national surveillance study. Br J Psychiatry 2011; 198: 295-301.

3 Hudson LD, Court AJ. What paediatricians should know about eating disorders in children and young people. J Paediatr Child Health 2012; Mar 7. doi: 10.1111/j.1440-1754.2012.02433.x. (Epub ahead of print)

4 Chui HT, Christensen BK, Zipursky RB, Richards BA, Hanratty MK, Kabani NJ, et al. Cognitive function and brain structure in females with a history of adolescent-onset anorexia nervosa. Pediatrics 2008; 122: e426-37.

5 Junior MARSIPAN Group. Junior MARSIPAN: Management of Really Sick Patients under 18 with Anorexia Nervosa (College Report CR168). RCPsych Publications, 2012.

Lee Hudson, Research Fellow and Honorary Consultant Paediatrician, Department of General and Adolescent Paediatrics, UCL Institute of Child Health, London, UK, email: I.hudson@ucl.ac.uk. Dasha Nicholls, Consultant Adolescent and Child Psychiatrist, Feeding and Eating Disorders Team, Great Ormond Street Hospital, London, UK. Debra Katzman, Professor of Paediatrics, Division of Adolescent Medicine, The Hospital for Sick Children and University of Toronto, Canada. Andrew Kennedy, Consultant Paediatrician, Department of Paediatric and Adolescent Medicine, Princess Margaret Hospital, Perth, Australia.

doi: 10.1192/pb.36.11.437

Author's reply: I fully agree with the authors that a careful definition of context is all important. So it was disappointing that they took my comment on being reassured out of the context in which it was placed; every death of a teenager is a tragedy. To note that the observed annual death rate for anorexia nervosa is around a tenth of that for the paediatric conditions they cite, and far less than the rate of death by suicide in young patients with schizophrenia, is not to be relaxed about risk in anorexia nervosa, nor to imply that specialised treatment for anorexia nervosa is not required.

In my response to Robinson's article I did not sufficiently well position my own observation about medical complications, which would have been better phrased as 'the only common complication of clinical significance'. Morbidity, which I was seeking to distinguish from the biological response to starvation, sometimes goes beyond bone mineralisation problems, and often does when chronicity becomes established. However, unlike Wentz et al's prospective outcome study, which reported no deaths over an 18-year 